\title{
Pain Physician Continues to Progress Towards Excellence
}

\section{Curtis W. Slipman, MD}

"With the affairs of active human beings it is different. Here knowledge of truth alone does not suffice; on the contrary this knowledge must continually be renewed by ceaseless effort, if it is not to be lost. It resembles a statue of marble which stands in the desert and is continuously threatened with burial by the shifting sands. The hands of science must ever be at work in order that the marble column continue everlastingly to shine in the sun. To those serving hands mine also belong."

Albert Einstein

The content and scope of the articles contained in this issue in many ways is a reflection of Einstein's quote about the pursuit of the knowledge of truth. We have included manuscripts that advance and refine our fundamental knowledge about clinical pain medicine. You will find articles about radiology, spinal anatomy, radiculopathy, historical and the most recent information about governmental oversight that will impact each reader of Pain Physician, and two papers concerning the treatment of low back pain.

The inaugural section of "Radiology Corner" can be found in this issue of Pain Physician. Isaac et al offer a focused discussion of the imaging characteristics of an interesting patient presenting with low back pain. Their discussion about this case meet the three criteria we have established for that section; all publishable submissions will include an imaging study that raises concerns about the differential diagnosis, a detailed explanation of the imaging findings, and an educational review of a relevant topic. While this was an invited article necessary to kick off "Radiology Corner," we expect the selection process to change with time. In order for such a change to transpire each reader is encouraged to send us a manuscript of a case they have

From Pain Physician. Dr. Slipman, the Editor-in-chief of Pain Physician, is director at Penn Spine Center and associate professor of Departments of Rehabilitation Medicine at University of Pennsylvania Medical Center. Address correspondence: Curtis W. Slipman, MD, The Penn Spine Center, Ground Floor White Bldg., 3400 Spruce Street, Philadelphia, PA 19104. E-mail: slipman@mail.upenn.edu found fascinating. Of course, as with all submissions, the peer review process will be implemented. Continuing with this radiologic theme, Fortin et al review one of the typical dilemmas confronted by pain physicians; which imaging study should I order for the patient with cervical radicular pain? By way of literature review they delve into the inherent abilities of various imaging modalities, costs, and study design limitations. You will also find an article on randomization in research design which raises many interesting and important questions. And finally, for the first time in the United States, an evaluation of the prevalence of cervical facet joint pain in chronic neck pain is being published in this issue. We are sure all of these articles will enhance your ability to practice pain medicine.

Slipman et al developed a manuscript that provides clinical data that implicates the presence of the repeatedly theorized process of chemical radiculitis. In that article they raise an interesting question of whether chemical radicular pain is an entity that occurs more frequently than generally appreciated. We anticipate this manuscript will raise questions that will merit further study. Another original contribution that will generate much thought is the work of Singh et al. Their study assesses the outcomes of patients with chronic low back pain, who undergo percutaneous decompression by Coblation technology. To date, this represents the first peer reviewed paper analyzing the results of this particular patient population. By way of reminder, to underscore our commitment to providing our readership with kind of information needed to practice cutting edge pain medicine, our last issue brought you the first study about Coblation technology for patients with back and/or leg pain. In my view these two papers provide compelling data supporting the prudent use of percutaneous disc decompression by Coblation for patients manifesting symptoms of back and/or leg pain from a contained focal protrusion. Certainly, additional well designed studies are needed before a definitive judgment can be proffered. Let me re-emphasize that by securing these manuscripts Pain Physician has singularly enabled interventional pain physicians to practice cutting edge medicine. By reporting upon the direction this novel approach is taking interventionalists can make a more informed decision about whether to incorporate this technique in their practice. 
Rosenberg et al provide their results following the use of transforaminal injections to treat the sequelae of discogenic disease. Though prior reports establish the value of transforaminal steroid placement for patients with radicular pain' there are no studies published that specifically address the patient population who only have axial complaints.

Manchikanti et al lead us in a very different, but important and revealing direction; government scrutiny and regulation. The first article reviews the National All Schedules Reporting Act (NASPER). This extensive and deeply researched document provides our readers with a keen sense of the scope of the issue and the potential implications to patients and pain physicians. It is a must read document as is the subsequent article about fraud and abuse. In this fascinating review we learn about current statutes, potential penalties, and the direction oversight agencies are taking.
As I mentioned in my first editorial, it is our goal to bring you the most up to date information about a variety of topics concerning interventional pain physicians through a peer reviewed format. I believe you will agree with me that we met that goal with this issue. As well, I intimated that we would include some changes to Pain Physician that will enhance its educational value. Our initial step was a series of detailed reviews of spinal anatomy articles, which is continued in this issue. Our second addition is the section "Radiology Corner". While the anatomy articles are and will be primarily, though not exclusively, invited reviews, it is our expectation that future articles in "Radiology Corner" will be generated from your submissions. The editorial board and I continue to work diligently to provide a product of which we can be proud. We look forward to your comments, critiques, and submissions to help us in our pursuit of excellence. 


\section{Pain Physician Continues to Progress Towards Excellence}

\section{Curtis W. Slipman, MD}

"With the affairs of active human beings it is different. Here knowledge of truth alone does not suffice; on the contrary this knowledge must continually be renewed by ceaseless effort, if it is not to be lost. It resembles a statue of marble which stands in the desert and is continuously threatened with burial by the shifting sands. The hands of science must ever be at work in order that the marble column continue everlastingly to shine in the sun. To those serving hands mine also belong."

Albert Einstein

The content and scope of the articles contained in this issue in many ways is a reflection of Einstein's quote about the pursuit of the knowledge of truth. We have included manuscripts that advance and refine our fundamental knowledge about clinical pain medicine. You will find articles about radiology, spinal anatomy, radiculopathy, historical and the most recent information about governmental oversight that will impact each reader of Pain Physician, and two papers concerning the treatment of low back pain.

The inaugural section of "Radiology Corner" can be found in this issue of Pain Physician. Isaac et al offer a focused discussion of the imaging characteristics of an interesting patient presenting with low back pain. Their discussion about this case meet the three criteria we have established for that section; all publishable submissions will include an imaging study that raises concerns about the differential diagnosis, a detailed explanation of the imaging findings, and an educational review of a relevant topic. While this was an invited article necessary to kick off "Radiology Corner," we expect the selection process to change with time. In order for such a change to transpire each reader is encouraged to send us a manuscript of a case they have

From Pain Physician. Dr. Slipman, the Editor-in-chief of Pain Physician, is director at Penn Spine Center and associate professor of Departments of Rehabilitation Medicine at University of Pennsylvania Medical Center. Address correspondence: Curtis W. Slipman, MD, The Penn Spine Center, Ground Floor White Bldg., 3400 Spruce Street, Philadelphia, PA 19104. E-mail: slipman@mail.upenn.edu found fascinating. Of course, as with all submissions, the peer review process will be implemented. Continuing with this radiologic theme, Fortin et al review one of the typical dilemmas confronted by pain physicians; which imaging study should I order for the patient with cervical radicular pain? By way of literature review they delve into the inherent abilities of various imaging modalities, costs, and study design limitations. You will also find an article on randomization in research design which raises many interesting and important questions. And finally, for the first time in the United States, an evaluation of the prevalence of cervical facet joint pain in chronic neck pain is being published in this issue. We are sure all of these articles will enhance your ability to practice pain medicine.

Slipman et al developed a manuscript that provides clinical data that implicates the presence of the repeatedly theorized process of chemical radiculitis. In that article they raise an interesting question of whether chemical radicular pain is an entity that occurs more frequently than generally appreciated. We anticipate this manuscript will raise questions that will merit further study. Another original contribution that will generate much thought is the work of Singh et al. Their study assesses the outcomes of patients with chronic low back pain, who undergo percutaneous decompression by Coblation technology. To date, this represents the first peer reviewed paper analyzing the results of this particular patient population. By way of reminder, to underscore our commitment to providing our readership with kind of information needed to practice cutting edge pain medicine, our last issue brought you the first study about Coblation technology for patients with back and/or leg pain. In my view these two papers provide compelling data supporting the prudent use of percutaneous disc decompression by Coblation for patients manifesting symptoms of back and/or leg pain from a contained focal protrusion. Certainly, additional well designed studies are needed before a definitive judgment can be proffered. Let me re-emphasize that by securing these manuscripts Pain Physician has singularly enabled interventional pain physicians to practice cutting edge medicine. By reporting upon the direction this novel approach is taking interventionalists can make a more informed decision about whether to incorporate this technique in their practice. 
Rosenberg et al provide their results following the use of transforaminal injections to treat the sequelae of discogenic disease. Though prior reports establish the value of transforaminal steroid placement for patients with radicular pain' there are no studies published that specifically address the patient population who only have axial complaints.

Manchikanti et al lead us in a very different, but important and revealing direction; government scrutiny and regulation. The first article reviews the National All Schedules Reporting Act (NASPER). This extensive and deeply researched document provides our readers with a keen sense of the scope of the issue and the potential implications to patients and pain physicians. It is a must read document as is the subsequent article about fraud and abuse. In this fascinating review we learn about current statutes, potential penalties, and the direction oversight agencies are taking.
As I mentioned in my first editorial, it is our goal to bring you the most up to date information about a variety of topics concerning interventional pain physicians through a peer reviewed format. I believe you will agree with me that we met that goal with this issue. As well, I intimated that we would include some changes to Pain Physician that will enhance its educational value. Our initial step was a series of detailed reviews of spinal anatomy articles, which is continued in this issue. Our second addition is the section "Radiology Corner". While the anatomy articles are and will be primarily, though not exclusively, invited reviews, it is our expectation that future articles in "Radiology Corner" will be generated from your submissions. The editorial board and I continue to work diligently to provide a product of which we can be proud. We look forward to your comments, critiques, and submissions to help us in our pursuit of excellence. 